\title{
MODELS OF SATURN'S RINGS WHICH SATISFY THE OPTICAL OBSERVATIONS*
}

\author{
Y. KAWATA and W. M. IR VINE \\ Dept. of Physics and Astronomy, University of Massachusetts, Amherst, Mass., U.S.A.
}

\begin{abstract}
A theoretical model of Saturn's rings is investigated which includes the shadowing effect and realistic anisotropic phase functions for the ring particles. The effects of multiple scattering and the finite size of the Sun, including the penumbra, are rigorously included. The permissible range of the relevant parameters, including optical thickness, single scattering albedo, volume density, and phase function are investigated by comparing the theoretical results to observations of the ring brightness vs phase angle, wavelength, and elevation of the Sun and Earth. Anisotropic scattering by the ring particles is necessary in order to match the observations. The colour dependence of the opposition effect is interpreted in terms of the albedo spectrum of the ring particles.
\end{abstract}

\section{Introduction}

The data provided by optical observations has traditionally been the core material for attempts to understand the nature of Saturn's rings. Although critical observations are now becoming available in the infrared and microwave regions of the spectrum, models of Saturn's rings must continue to satisfy the constraints provided by the optical data.

By optical in the present context we mean the extended visible portion of the spectrum (roughly $0.3-1.0 \mu$ ) in which the radiation received at the Earth from Saturn's rings is reflected sunlight, and hence does not include thermal emission by the ring particles. Previous analyses of the optical observations have suffered from limitations which are no longer necessary in view of improved computational and theoretical methods. We have accordingly endeavored to apply the best procedures currently available in an effort to see what limitations are imposed upon the physical parameters of the ring system and the particles which it contains.

The procedure which we shall use is a refinement of that originally proposed by Seeliger (1887) and subsequently employed in the fundamentally important work of Bobrov (e.g., 1970) and Franklin and Cook (1965).

\section{Available Observations and Outline of the Procedure}

Since the most complete and reliable photometric data are available only for the bright ring $B$, we shall concentrate our attention on this ring. The fundamental observation which must be matched by any theoretical model is the phase curve of the rings; that is, the surface brightness normalized at phase angle 0 vs phase angle $\alpha$. This curve is normally plotted in stellar magnitudes per square arc-second of the rings, and has the following three characteristic features:

\footnotetext{
* Contribution Number 172 from the Five College Observatories.
} 
(a) A very sharp surge in brightness near $\alpha=0$ which is known as the opposition effect;

(b) A linearly decreasing brightness as $\alpha$ increases for $\alpha \gtrsim 2^{\circ}$;

(c) A dependence upon wavelength.

The most reliable photometric phase curves appear to be those of Franklin and Cook (1965) which were obtained in the $B$ and $V$ wavelength bands. This data is illustrated in Figure 1. Since Saturn did not reach exact opposition $(\alpha=0)$ during their observations and the extrapolation to $\alpha=0$ is somewhat arbitrary, we have normalized all data at the minimum phase angle observed $\left(\alpha=0.094^{\circ}\right)$.

Another observation of critical importance to the understanding of the rings is the absolute surface brightness as a function of wavelength. Data for $B$ and $V$ were obtained by Franklin and Cook (1965). Corresponding data for other wavelengths may. be obtained from the relative spectral photometry of Lebofsky et al. (1970), Irvine and Lane (1973), and Kharitonova and Teifel (1973), although care must be taken to insure that observations made under corresponding conditions are compared. This is important because the brightness of the rings may depend upon a number of geometric factors, including $\alpha$ and declination of the Sun and Earth relative to the ring plane, as well as possibly on distance of Saturn from the Sun or the position of the ring particles relative to their eclipse by Saturn.

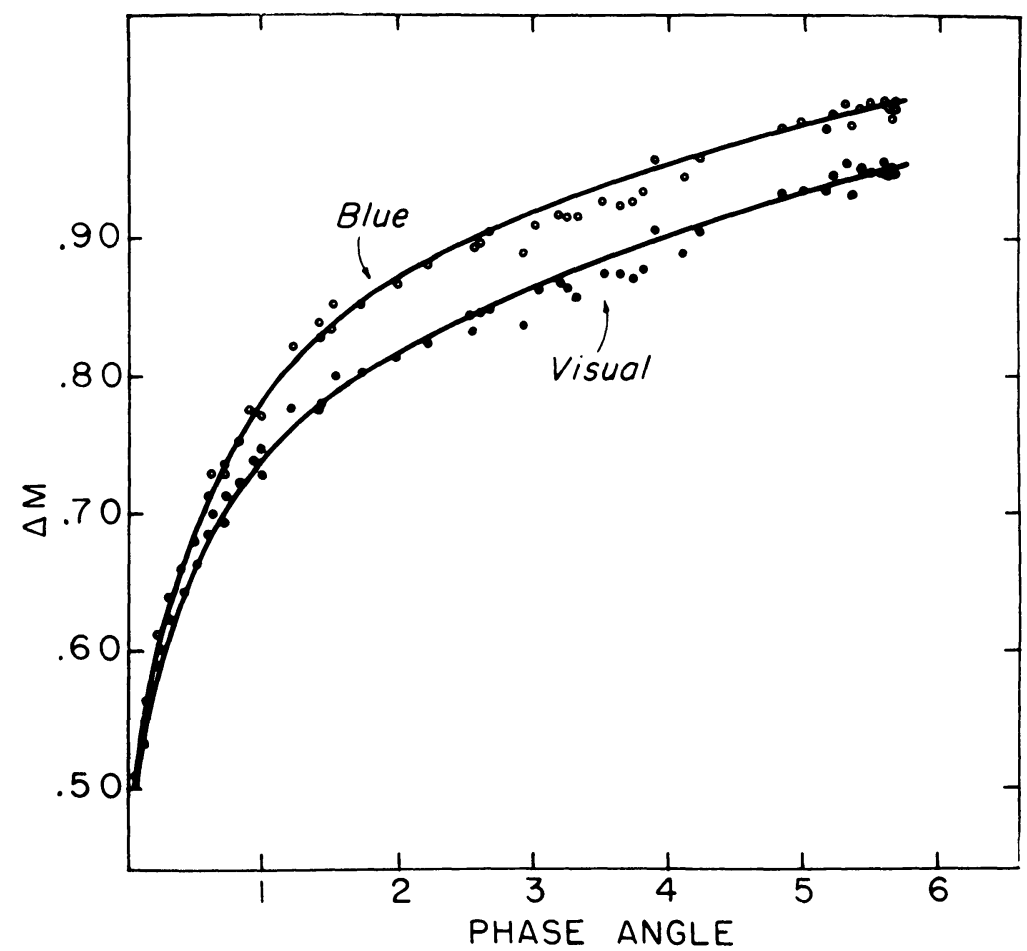

Fig. 1. Phase curves for ring $B$ at two wavelengths normalized at $x=0.094$ (from Franklin and Cook, 1965). 
Additional photometric observations of potential importance are the variation in ring brightness with declination of the Sun and Earth. Observations have been made by Camichel (1958) and Price (1973). Complete phase curves of the rings at wavelengths in the red and near ultraviolet would be most desirable in the future.

The principle diagnostic characteristic of the phase curve is the opposition effect. We shall procede on the assumption that this effect is produced by the mutual shadowing of the ring particles, an idea originally proposed by Seeliger (1887). In more detail, we assume that the rings consist of a plane layer containing many independent particles which are illuminated by the Sun and observed from the Earth. Those particles nearer the Sun cast shadows upon the particles behind. At exact opposition an observer on Earth will see only sunlit particles and so will observe a maximum surface brightness. As the phase angle increases, the shadowed particles which were formerly shielded from view by the sunlit particles may now be observed from the Earth, so that the surface brightness falls off. This initial decrease in brightness takes place very rapidly as a function of phase angle.

Observations of stellar occultations by Saturn's rings indicate that the optical thickness of ring B is near unity. Because of the relatively high albedo of the ring particles (see below), it then follows that multiple scattering will play an important role in determining the photometric properties of the ring system. In previous computations of the shadowing mechanism multiple scattering has been included in only an approximate manner, by assuming that higher order scattering will be isotropic. In the present paper we treat rigorously the multiple scattering problem for more realistic, anisotropic particle phase functions.

The close relationship between the shadowing mechanism described above and the usual multiple scattering theory of radiative transfer has been discussed by Irvine (1966). Radiative transfer theory can be applied when the interparticle distance in the layer is sufficiently large that each particle is effectively in the far field for scattering by the other particles, so that shadows may be neglected. When the particles are large enough and their number density is great enough, they will cast shadows upon each other, and the usual multiple scattering theory must be modified to include the effect of shadowing. Fortunately, this can be done in a straight-forward manner.

\section{Theoretical Procedure}

We shall assume in our model that the rings are plane-parallel and homogeneous with respect to optical depth. We thus neglect the possibility that such properties as mean particle size or composition depend on altitude with respect to the center of the ring plane. We shall furthermore assume for the present that the ring particles may be characterized by a single effective radius $\varrho$ and for the purposes of the shadowing computation may be treated as spheres. We shall return in Section 5 below to the possibility of a distribution of particle sizes, which introduces into the theory such quantities as $\left\langle\varrho^{2}\right\rangle^{1 / 2}$ and $\left\langle\varrho^{3}\right\rangle^{1 / 3}$ in addition to the mean radius $\varrho$. The assumption of sphericity will not significantly effect the applicability of our results, since it can be 
shown that the magnitude of the shadowing effect at opposition is independent of the particle shape (Seeliger, 1895), and we do not require that the individual particle phase function be given by Mie theory.

Following the procedure of Irvine (1966), we may express the specific intensity $I$ of the radiation reflected by the rings as a sum of successive orders of scattering:

$$
I=I_{1}^{s}+\sum_{n=2}^{\infty} I_{n} a^{n}
$$

where $I_{1}^{s}$ is the contribution from once-scattered radiation including the necessary shadowing correction, and $I_{n} a^{n}$ is the contribution from radiation scattered $n$ times. Fortunately, the effect of mutual shadowing is important only in the calculation of the primary scattered intensity and may be neglected in the computation of $I_{n}$ for $n \geqslant 2$.

\subsection{Shadowing MECHANISM}

Let us discuss first the computation of $I_{1}^{s}$. If the wavelength of light $\lambda$ is such that

$$
\lambda \ll \varrho^{2} / \Delta,
$$

where $\Delta$ is the lesser of the mean free-path of a photon in the layer and the thickness of the layer, a shadow will be formed behind each particle which will be described by geometric optics (van de Hulst, 1957). Let us introduce coordinates such that $\theta=$ $=\arccos \mu$ is the polar angle with respect to the outward normal to the ring layer and $\phi$ is the corresponding azimuthal angle measured from the plane of incidence. We shall use the notation $\Omega=(\theta, \phi)$ to specify a particular direction, and shall initially assume that solar radiation is incident only in the direction $\Omega_{0}=\left(\theta_{0}, \phi_{0}\right)$. For convenience we take $\mu_{0} \equiv\left|\cos \theta_{0}\right|$. Let the thickness of the ring layer be $t$, and let the fraction of the ring volume occupied by particles be $D$, so that

$$
D=\frac{4}{3} \pi \varrho^{3} n,
$$

where $n$ is the number density of particles in the rings.

The physical mechanism operative in the shadowing effect can be understood by referring to Figure 2 which shows a particle of radius $\varrho$ at a depth $h$ within the ring layer. The volumes $V_{1}$ and $V_{2}$ are (except for a small correction near the surface) cylinders of base area $\pi \varrho^{2}$ and height $h / \mu_{0}$ and $h / \mu$, respectively. A small element $\varepsilon$ of the projected area of the particle will be both sunlit and observable from the Earth provided that the centers of all other particles in the ring layer are outside of the volumes $V_{1}$ and $V_{2}$. If, as implied by our postulate of homogeneity, the ring particles are randomly distributed through the ring volume, the probability of the above situation occurring may be easily computed upon the assumption that the fractional volume occupied by particles is sufficiently small $(8 D \ll 1)$. This probabilistic approach yields the familiar exponential attenuation for both the average radiation field at a depth $h$ in the layer and for the primary scattered radiation emerging from the layer, provided that the volumes $V_{1}$ and $V_{2}$ do not significantly overlap. If they do so overlap, 


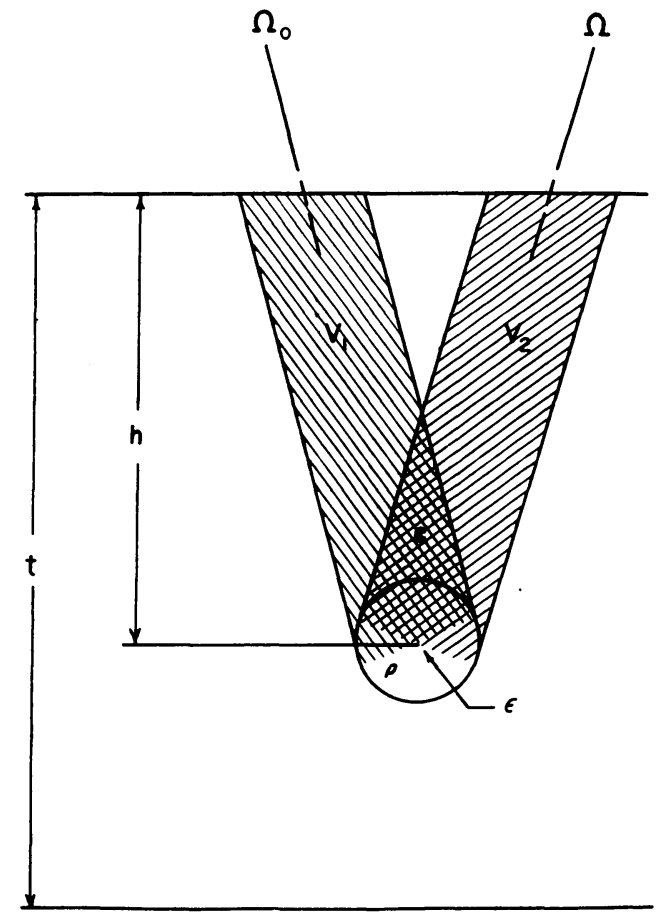

Fig. 2. Geometry of the shadowing effect. Direction towards the Sun is $\Omega_{0}$, towards the Earth is $\Omega$.

an anomalously high intensity is produced because the probability for photon escape from a depth $h$ becomes highly correlated with the probability of photon penetration to the same point. This is the shadowing effect.

The analysis shows that, if the incident solar flux through the upper surface is $\pi$, the single-scattered intensity at an optical depth $\zeta=n \pi \varrho^{2} h$ is given by

$$
I_{1}^{s}=\frac{a \Phi\left(\Omega, \Omega_{0}\right)}{4 \mu \mu_{0}} e^{\zeta / \mu} \int_{\zeta}^{\tau} \mathrm{d} \zeta^{\prime} \exp \left[-\zeta^{\prime}\left(\mu^{-1}+\mu_{0}^{-1}\right)+n C\right],
$$

where $n$ is the number density of particles, $C$ is the overlap volume shown in Figure 2, $a$ is the single-scattering albedo of the ring particles, the particle phase function is $\Phi$, and the polar angles of incident and scattered light $\arccos \mu_{0}$ and $\arccos \mu$ are measured from the outward normal. Clearly, when $C=0, I_{1}^{s}$ is the primary scattering obtained from the usual multiple scattering theory. Since we have assumed that the particles are large and diffraction may be neglected (that is, the efficiency factor for extinction is unity), the optical thickness of the rings is related to the parameters previously introduced by

$$
\tau=n \pi \varrho^{2} t
$$


The quantity $n C$ has the form (Irvine, 1966)

where

$$
n C= \begin{cases}v_{1} & \left(\zeta^{\prime}-\zeta\right) \geqslant Z, \quad \alpha \neq 0 \\ v_{1}-\Sigma & \left(\zeta^{\prime}-\zeta\right) \leqslant Z, \quad \alpha=0 \\ \left(\zeta^{\prime}-\zeta\right) / \mu_{0} & (\alpha=0),\end{cases}
$$

$$
\begin{aligned}
v_{1} & =\frac{(1+\cos \alpha) D}{\pi \sin \alpha} \\
\Sigma \quad & =\frac{3\left(\cos \theta+\cos \theta_{0}\right)^{2} D}{4 \pi \sin \alpha \cos \theta \cos \theta_{0} \cos v}\left[\cos \psi-\frac{\cos ^{3} \psi}{3}-(\pi / 2-\psi) \sin \psi\right] \\
\cos \alpha= & \cos \theta \cos \theta_{0}+\sin \theta \sin \theta_{0} \cos \left(\phi-\phi_{0}\right) \\
\cos \delta= & \left(\cos \theta_{0}-\cos \theta \cos \alpha\right) /(\sin \theta \sin \alpha) \\
\tan v= & \sin \theta \sin \delta \sin \alpha /\left(\cos \theta+\cos \theta_{0}\right) \\
\sin \psi= & \frac{4\left(\zeta^{\prime}-\zeta\right) \sin \alpha \cos v}{3\left(\cos \theta+\cos \theta_{0}\right) D} \\
Z \quad= & \frac{3\left(\cos \theta+\cos \theta_{0}\right)}{4 \quad \sin \alpha \cos v} .
\end{aligned}
$$

When $\zeta=0$, we find the reflected intensity as

$$
R_{1}^{s} \equiv I_{1}^{s}(\zeta=0) \quad(\mu>0)
$$

The above approach is essentially that used by Seeliger (1887). Because of the geometry in Figure 2, it is referred to as the cylinder-cylinder model. Bobrov (cf. 1970) has pointed out the important effect introduced by the finite angular diameter of the Sun at the distance of Saturn. He modified the previous theory by replacing the volume $V_{1}$ in Figure 2 by a conical volume, producing a 'cone-cylinder model'. This procedure, however, ignores the penumbra of the shadows cast.

Franklin and Cook (1965) observed that the opposition effect appears to be wavelength dependent, and proposed a model in which this dependence was produced by the wavelength variation of diffraction into the shadow zone. They treated this situation by using a 'cone-cone' model for the shadowed volumes, with the dimensions of the 'diffraction cone' being wavelength dependent. This model led to an unreasonably small physical thickness of the ring, however, and it is desirable to search for an alternative mechanism for producing the wavelength dependence. Franklin and Cook also considered the possibility that the wavelength effect might be due to differences in the glory produced by small Mie-scattering spheres forming a surface structure on larger particles. This seems extremely unlikely, however, because of the high degree of symmetry of the scattering centers needed to produce the glory phenomenon.

In the present model we propose to take into account the effect of the Sun's finite size by performing a numerical average of the intensity obtained under the assumption of a point (infinitely distant) Sun. This will rigorously include the effect of the penumbra and also any influence of solar limb darkening. The computation can be 
carried out quite rigorously, because the necessary arithmetic in Equations (3) and (5) is efficiently and rapidly performed on an electronic computer. The resulting model will have a reduced opposition effect relative to the point Sun model, because there is no longer an exact opposition for the total solar flux.

If the angular diameter of the Sun at Saturn's distance is $\beta$ (about $3^{\prime}$ ), and the Sun is assumed symmetric about the angular direction $\Omega_{00}=\left(\theta_{v u}, 0\right)$ of its midpoint, the average reflected intensity in the direction $(\theta, \phi)$ will be

$$
\left\langle R_{1}^{s}(\theta, \phi)\right\rangle \equiv \frac{\int_{0}^{\beta / 2} \mathrm{~d} \theta^{\prime} \int_{0}^{2 \pi} \mathrm{d} \phi^{\prime} \sin \theta^{\prime} W\left(\cos \theta^{\prime}\right) R_{1}^{s}\left[\theta, \phi ; \theta_{0}\left(\Omega^{\prime}\right), \phi_{0}\left(\Omega^{\prime}\right)\right] \cos \theta_{0}\left(\Omega^{\prime}\right)}{\int_{0}^{\beta / 2} \mathrm{~d} \theta^{\prime} \int_{0}^{2 \pi} \mathrm{d} \phi^{\prime} \sin \theta^{\prime} W\left(\cos \theta^{\prime}\right) \cos \theta_{0}\left(\Omega^{\prime}\right)},
$$

where the solar limb darkening

$$
\begin{aligned}
W\left(\mu^{\prime}\right) & =a_{\lambda}+b_{\lambda} \mu^{\prime}+c_{\lambda}\left[1-\mu^{\prime} \ln \left(1+\left(\mu^{\prime}\right)^{-1}\right)\right] \\
\mu^{\prime} & =\cos \theta^{\prime}
\end{aligned}
$$

is taken from Pierce and Waddell (1961). The primed coordinates are measured with respect to the direction $\Omega_{00}$ as polar axis. The relevant angles may be obtained from spherical trigonometry as

$$
\begin{aligned}
& \cos \theta_{0}=\cos \theta^{\prime} \cos \theta_{00}-\sin \theta^{\prime} \sin \theta_{00} \cos \phi^{\prime} \\
& \sin \phi_{0}=\sin \theta^{\prime} \frac{\sin \phi^{\prime}}{\sin \theta_{0}} .
\end{aligned}
$$

The integrations were carried out by a Gaussian procedure using as many as 14 points in both $\theta$ and $\phi$. The results are quite insensitive to the particular limb darkening law chosen.

One measure of the theoretical amplitude of the mutual shadowing effect which provides some insight into the differences between the present model and previous ones is the magnitude difference $\delta M(D)$ between the primary scattered reflection at $\alpha=0$ (where the shadowing effect is maximum) and at $\alpha=6^{\circ}$ (where its effect is small):

$$
\delta M(D)=-2.5 \log \frac{\left[R_{1}^{s}\left(\alpha=6^{\circ}\right)\right]}{\left[R_{1}^{s}\left(\alpha=0^{\circ}\right)\right]} .
$$

We have written $\delta M(D)$, since the volume density $D$ is the principal parameter determining the strength of the shadowing effect. Figure 3 presents results of computations for $\delta M(D)$ for the case $\tau=1, \theta=\theta_{0}=64^{\circ}$ (which approximates the Saturnocentric declination of the Sun and Earth during the observations of Franklin and Cook), and $\Phi=1$. The notation 'point $\odot$ ' refers to results based on the cylinder-cylinder model described above; 'finite $\odot$ ' represents the refined cylindrical model which we shall employ and which is described by Equation (7); and 'Bobrov' refers to the conecylinder model of that author (see Bobrov, 1970).

For both the point $\odot$ and the finite $\odot$ models the shadowing effect (neglecting 


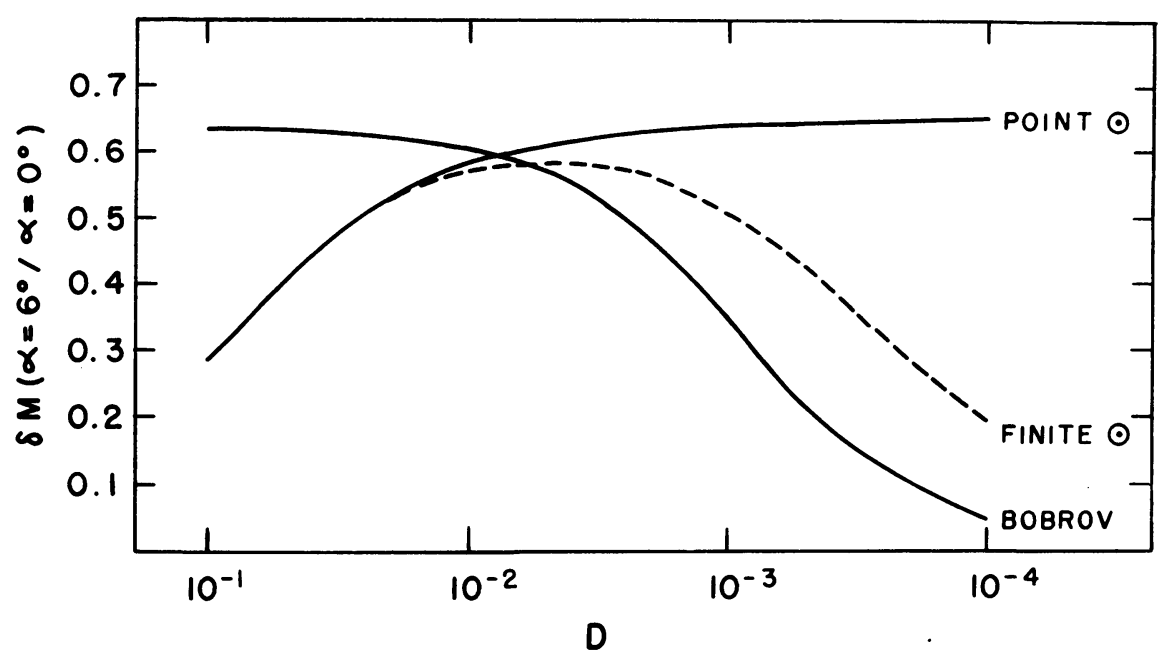

Fig. 3. Magnitude of the shadowing effect $\delta M(D)$ for first order scattering as a function of fractional volume $D$ occupied by ring particles. Geometry appropriate to Franklin and Cook (1965) data, and $\tau=1$. See Equation (8).

multiple scattering) decreases for large volume densities because the surface of the rings appears to become smoother (uniformly filled). Because of certain simplifying assumptions in the mathematics, the Bobrov model does not exhibit this behavior. For very small values of $D$, the point $\odot$ model approaches an asymptotic value for $\delta M$; this behavior is a result of the infinite extent of the cylindrical shadows, and the fact that for a given optical depth $\tau$, as $D$ decreases, the thickness of the layer $t$ must increase (see Equation (4)). In contrast, both the finite $\odot$ and Bobrov models produce shadows of finite length, so that for sufficiently small $D$ (sufficiently large interparticle distance) the shadowing effect vanishes. The Bobrov model produces a smaller shadowing effect for small $D$ because of inaccuracies in its treatment of the penumbra.

A more detailed view of the relation between $\delta M$ and $D$ may be obtained by considering the entire phase curve $M_{1}(\alpha)$ as a function of $D$. Sample phase curves for various $D$ computed from our shadowing theory are shown in Figure 4 for the same parameters used in Figure 3. We note the following points:

(a) The smaller the volume density $D$, the steeper the initial decrease in brightness with increasing phase angle (that is, the more peaked is the opposition effect).

(b) For $D \lesssim 0.01$, the total shadowing effect $\delta M$ over the phase angle range $0-6^{\circ}$ increases as $D$ increases.

(c) For $D \gtrsim 0.01$, the opposition effect has become so broad that $\delta M$ begins to decrease although the phase curve is still falling off at $6^{\circ}$. We should point out that the values of $\delta M$ in Figure 4 differ from those in Figure 3 for the same $D$ because in the former case they have been normalized at $\alpha=0.094^{\circ}$ for comparison with the data of Franklin and Cook, while in the latter case the normalization was at exact opposition $(\alpha=0)$. 


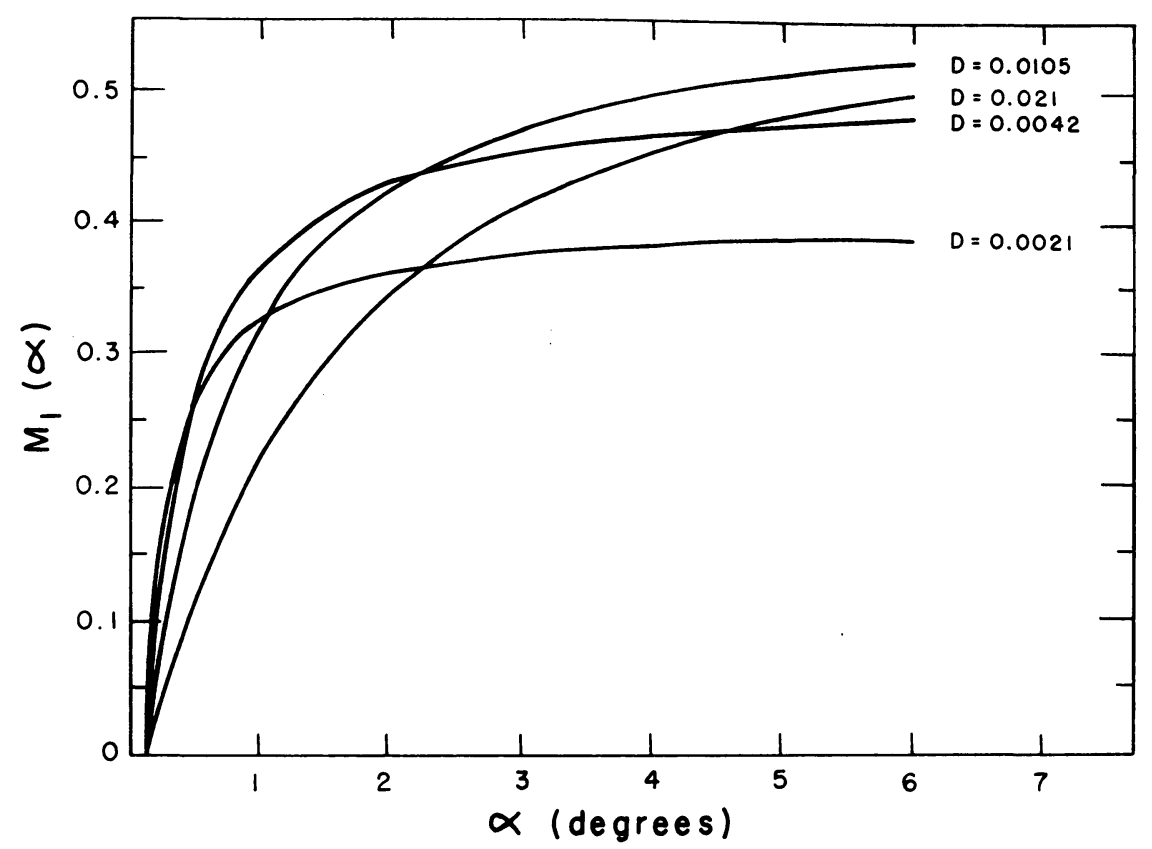

Fig. 4. Theoretical phase curves for first order scattering with $\tau=1, \theta=\theta_{0}=64^{\circ}$. Curves labeled with the density parameter $D$.

In fact none of the curves presented in Figure 4 agrees with the data (cf. Figure 1). If the theoretical curves are sufficiently steep for small phase angles, they are too flat for $\alpha \gtrsim 2^{\circ}$. We therefore must consider the additional effects of the particle phase function $\Phi$ and of higher order scattering (next section). Note, however, that since the sharp opposition peak is primarily the result of the shadowing mechanism and not these other effects, we may say from a comparison of Figures 1 and 4 that $D \lesssim 0.02$ if we are to produce a sufficiently sharp peak.

\subsection{Multiple SCATtering}

It seems to us reasonable to suppose that the wavelength dependence of the phase curve may be due largely to variation of the single-scattering albedo $a$ of the particles. The spectra obtained by Lebofsky et al. (1970) show that the ring reflectivity varies significantly between the ultraviolet and the infrared. Increasing the value of $a$ will significantly change the multiply scattered contribution to the reflected intensity, with a resulting dilution of the opposition effect and change in shape of the phase curve. Let us compute the magnitude of this effect.

Apart from the shadowing effect, the intensity reflected by Saturn's rings will satisfy the equation of radiative transfer

$$
\mu_{\mathrm{d} \zeta}^{\mathrm{d} I}=-I+\frac{a}{4 \pi} \int_{4 \pi} \mathrm{d} \Omega^{\prime} \Phi\left(\Omega, \Omega^{\prime}\right) I\left(\Omega^{\prime}\right),
$$


where $a$ and $\Phi$ have been defined above. Since the optical thickness of the rings is not large, it is convenient to express the solution to Equation (9) as the sum of successive orders of scattering (van de Hulst, 1948; van de Hulst and Irvine, 1963; Irvine, 1964), so that

$$
I(\Omega, \zeta)=\sum_{n=0}^{\infty} a^{n} I_{n}(\Omega, \zeta)
$$

where $I_{n}$ is the $n$th order intensity for $a=0$. Since we take the optical thickness of the rings as known in the computation of a particular model, Equation (10) provides an efficient means for determining the effect of a change in particle albedo. The desired ring reflectivity including the opposition effect is thus, from Equations (1), (6), and (10),

$$
R(\Omega)=R_{1}^{s}(\Omega)+I(\Omega, 0)-a I_{1}(\Omega, 0) \quad(\mu>0) .
$$

The ring reflectivity will be a function of the parameters $a, \tau, \Omega_{0}$, and $D$, as well as direction $\Omega$ and the properties of the phase function $\Phi$.

The successive terms in Equation (10) are found, for incident flux $\pi$ through the horizontal upper boundary of the rings, from the relations

$$
\begin{aligned}
& I_{0}(\Omega, \zeta)=\frac{\pi \delta\left(\mu-\mu_{0}\right) \delta\left(\phi-\phi_{0}\right) e^{-\zeta / \mu_{0}}}{\mu_{0}} \\
& B_{n}(\Omega, \check{\zeta})=\frac{1}{4 \pi} \int_{0}^{2 \pi} \mathrm{d} \phi^{\prime} \int_{-1}^{1} \mathrm{~d} \mu^{\prime} \Phi\left(\Omega, \Omega^{\prime}\right) I_{n-1}\left(\Omega^{\prime}, \zeta\right) \\
& I_{n}^{-}(\Omega, \zeta)=\int_{0}^{\zeta} \mathrm{d} \zeta^{\prime} e^{-\left(\zeta^{\prime}-\zeta\right) / \mu} \frac{B_{n}\left(\Omega, \zeta^{\prime}\right)}{-\mu} \\
& I_{n}^{+}(\Omega, \zeta)=\int_{\zeta}^{\tau} \mathrm{d} \zeta^{\prime} e^{-\left(\zeta^{\prime}-\zeta\right) / \mu} \frac{B_{n}\left(\Omega, \zeta^{\prime}\right)}{\mu} \\
& I_{n}(\theta=\pi / 2, \phi, \zeta)=B_{n}(\theta=\pi / 2, \phi, \zeta)
\end{aligned}
$$

where $B_{n}$ is the so-called source function for $n$th order scattering and the superscripts + and - refer to the cases $\theta<\pi / 2$ and $\theta>\pi / 2$, respectively. As has been emphasized in the above references, the ratio of successive terms $I_{n} / I_{n-1}$ approaches a constant value as $n$ increases, so that the series (10) may be truncated and the remainder replaced by a geometric series.

The double integrations over $\theta$ and $\phi$ in the above equations may be eliminated by expanding the phase function in a cosine series in $\left(\phi-\phi_{0}\right)$ (e.g., Hansen, 1969). Setting

$$
\Phi\left(\mu, \phi ; \mu, \phi_{0}\right)=\sum_{m=0}^{\infty} \Phi^{m}\left(\mu, \mu_{0}\right) \cos m\left(\phi-\phi_{0}\right),
$$


we have

where

$$
\begin{aligned}
& B_{n}\left(\mu, \phi, \zeta ; \mu_{0}, \phi_{0}\right)=\sum_{m=0}^{\infty} B_{n}^{m}\left(\mu, \zeta ; \mu_{0}\right) \cos m\left(\phi-\phi_{0}\right) \\
& I_{n}^{+}\left(\mu, \phi, \zeta ; \mu_{0}, \phi_{0}\right)=\sum_{m=0}^{\infty} I_{n}^{m+}\left(\mu, \zeta ; \mu_{0}\right) \cos m\left(\phi-\phi_{0}\right) \\
& I_{n}^{-}\left(\mu, \phi, \zeta ; \mu_{0}, \phi_{0}\right)=\sum_{m=0}^{\infty} I_{n}^{m-}\left(\mu, \zeta ; \mu_{0}\right) \cos m\left(\phi-\phi_{0}\right),
\end{aligned}
$$

$$
\begin{aligned}
& B_{1}^{m}=\frac{1}{4 \mu_{0}} e^{-\zeta / \mu_{0}} \Phi^{m}\left(\mu, \mu_{0}\right) \\
& B_{n}^{m}=\frac{1}{\left(4-2 \delta_{0, m}\right)} \int_{-1}^{1} \mathrm{~d} \mu^{\prime} \Phi^{m}\left(\mu, \mu^{\prime}\right) I_{n-1}^{m}\left(\zeta, \mu^{\prime}, \mu_{0}\right) \\
& I_{n}^{m-}\left(\mu, \zeta, \mu_{0}\right)=\int_{0}^{\zeta} \frac{\mathrm{d} \zeta^{\prime}}{-\mu} e^{-\left(\zeta^{\prime}-\zeta\right) / \mu} B_{n}^{m}\left(\mu, \zeta^{\prime}, \mu_{0}\right) \\
& I_{n}^{m+}\left(\mu, \zeta, \mu_{0}\right) \int_{\zeta 0}^{\tau} \frac{\mathrm{d} \zeta^{\prime}}{\mu} e^{-\left(\zeta^{\prime}-\zeta\right) / \mu} B_{n}^{m}\left(\mu, \zeta^{\prime}, \mu_{0}\right)
\end{aligned}
$$

Since the ring particle phase function is not known a priori, we shall choose a simple analytic expression which may be parameterized to conveniently describe a wide variety of phase functions. Such a function, which is also easily expressed in the form of Equation (13), is the Henyey-Greenstein function

where

$$
\Phi_{\mathrm{HG}}(\gamma, g)=\frac{\left(1-g^{2}\right)}{\left(1+g^{2}-2 g \cos \gamma\right)^{3 / 2}},
$$

$$
g=\frac{1}{2} \int_{-1}^{1} \mathrm{~d} \mu \mu \Phi(\mu) .
$$

We note that

$$
\Phi_{\mathrm{HG}}(\gamma, g)=\sum_{n}(2 n+1) g^{n} P_{n}(\cos \gamma)
$$

where

$$
\cos \gamma=\mu \mu_{0}+\sqrt{1-\mu^{2}} \sqrt{1-\mu_{0}^{2}} \cos \left(\phi-\phi_{0}\right)
$$

and $P_{n}$ and $P_{n}^{m}$ are the Legendre and the associated Legendre polynomials, respectively. Using the addition theorem we thus obtain

$$
\begin{array}{r}
\Phi_{\mathrm{HG}}(\gamma, g)=1+\sum_{n=1}^{\infty}(2 n+1) g^{n}\left[P_{n}(\mu) P_{n}\left(\mu_{0}\right)+2 \sum_{m=1}^{n} \frac{(n-m) !}{(n+m) !} \times\right. \\
\left.\times P_{n}^{m}(\mu) P_{n}^{m}\left(\mu_{0}\right) \cos m\left(\phi-\phi_{0}\right)\right]
\end{array}
$$


which may be written

where

$$
\Phi_{\mathrm{HG}}(\gamma, g)=\sum_{\kappa=0}^{\infty} \Phi_{\mathrm{HG}}^{\kappa}\left(\mu, \mu_{0}, g\right) \cos \kappa\left(\phi-\phi_{0}\right)
$$

$$
\begin{aligned}
& \Phi_{\mathrm{HG}}^{0}\left(\mu, \mu_{0}, g\right)=1+\sum_{n=1}^{\infty}(2 n+1) g^{n} P_{n}(\mu) P_{n}\left(\mu_{0}\right) \\
& \Phi_{\mathrm{HG}}^{\kappa}\left(\mu, \mu_{0}, g\right)=2 \sum_{n=\kappa}^{\infty}(2 n+1) g^{n} \frac{(n-\kappa) !}{(n+\kappa) !} P_{n}^{\kappa}(\mu) P_{n}^{\kappa}\left(\mu_{0}\right), \quad \kappa \neq 0 .
\end{aligned}
$$

In our models for this paper we shall use the phase function

$$
\Phi(\gamma)=b \Phi_{\mathrm{HG}}\left(\gamma, g_{1}\right)+(1-b) \Phi_{\mathrm{HG}}\left(\gamma, g_{2}\right)
$$

which is normalized such that

$$
\frac{1}{2} \int_{0}^{\pi} \mathrm{d} \gamma \sin \gamma \Phi(\gamma)=1
$$

Equation (22) allows us to investigate particle phase functions which are isotropic, principally forward directed, principally backward directed, or which contain both a forward and backward peak.

\section{Comparison with the Observations}

Using the results of the previous section we may write for the theoretically predicted phase curve $M(\alpha)$

$$
M(\alpha)=-2.5 \log \left[\frac{\left\langle R_{1}^{s}(\alpha)\right\rangle+\sum_{n=2}^{\infty} R_{n}(\alpha)}{\left\langle R_{1}^{s}(0)\right\rangle+\sum_{n=2}^{\infty} R_{n}(0)}\right],
$$

where we write $R_{n}=a^{n} I_{n}^{-}(\zeta=0)$ and the angular brackets denote an integration of the incident radiation over the disk of the Sun. In order to illustrate more clearly the role of the parameters involved, we may rewrite (24) as

$$
M(\alpha)=-2.5 \log \left[\frac{a \Phi(\pi-\alpha)\langle S(\alpha)\rangle+\sum_{n=2} R_{n}(\alpha)}{a \Phi(\pi)\langle S(0)\rangle+\sum_{n=2} R_{n}(0)}\right],
$$

where

$$
\langle S(\alpha)\rangle \equiv\left\langle\frac{1}{4 \mu \mu_{0}} \int_{0}^{\tau} \mathrm{d} \zeta^{\prime} \exp \left[-\zeta^{\prime}\left(\frac{1}{\mu}+\frac{1}{\mu_{0}}\right)+n C(\alpha)\right]\right\rangle
$$

is the primary scattered intensity including the shadowing effect for the case of conservative, isotropic scattering. 
Equation (25) may be further transformed to simplify the comparison with the observations. Because the higher order scattering component of the intensity does not change rapidly with angle and because the maximum phase angle observable for Saturn is $6^{\circ}$, we will have to a good approximation that

$$
\sum_{n=2}^{\infty} R_{n}(0) \simeq \sum_{n=2}^{\infty} R_{n}(\alpha)
$$

In fact, for the cases investigated below, Equation (27) holds to better than $1 \%$. It is then convenient to rewrite Equation (25) às

where

$$
M(\alpha)=-2.5 \log \left[\frac{\frac{\Phi(\pi-\alpha)\langle S(\alpha)\rangle}{\Phi(\pi)\langle S(0)\rangle}+x}{1+x}\right],
$$

$$
x \equiv \frac{\sum_{n=2}^{\infty} R_{n}(0)}{a \Phi(\pi)\langle S(0)\rangle}
$$

is the fraction of the intensity observed at exact opposition which is due to multiple scattering, while the other ratio in the numerator of Equation (28) is the intensity ratio which appeared in the definition of $\delta M(D)$. We thus see that the shape of the phase curve will depend on the quantities $x$, the phase function $\Phi$, and the optical thickness $\tau$ and volume density $D$ through $\langle S\rangle$. The single scattering albedo $a$ enters indirectly through $x$. It is these parameters $D, \tau, a$, and the quantities characterizing the phase function which we wish to determine.

In addition to the phase curve, the absolute surface brightness of ring B at opposition is a critical measurement for defining the ring parameters. Noting that the incident flux on the rings will be $\pi F \mu_{0}$, where $\pi F$ is the solar flux at the distance of Saturn through an area normal to the direction to the Sun, we may relate the observed absolute brightness at opposition to that intensity $R^{\circ} \equiv R(\alpha=0)$ calculated from Equation (11) by

$$
\begin{aligned}
R^{0} & =\frac{\pi I_{R}}{\pi F \mu_{0}} \frac{I_{D}}{I_{D}}=\frac{1}{\mu_{0}} \frac{I_{R}}{I_{D}} \frac{I_{D} / \pi F}{(1 / \pi)} \\
R^{0} & =\frac{1}{\mu_{0}} p_{\mathrm{S}} \frac{I_{R}}{I_{D}}
\end{aligned}
$$

where $I_{D}$ is the mean specific intensity averaged over Saturn's disk, $I_{R}$ is the mean specific intensity of the ring $\mathrm{B}$, and $p_{\mathrm{S}}$ is the geometric albedo of Saturn's disk. Using the data of Cook et al. (1973) for $I_{R} / I_{D}$ and the value $p_{\mathrm{S}}=0.429$ for $V$ from Irvine and Lane (1973), we find a value in the visual of $R_{V}^{0} \simeq 1.2$. Because the disk was partly shielded by the rings during the Franklin-Cook observations, this value of $R_{V}^{0}$ is based on an $I_{D}$ which will be biased towards Saturn's equatorial regions. We may obtain an independent estimate of the brightness of ring $B$ by multiplying the corresponding data of Price (1973), which apply to the total ring system, by a factor of 1.2, which is 
the correction determined from Franklin and Cook necessary to transform to ring B alone. The Price data give $R_{V}^{0}=1.1 \pm 0.1$ at a ring inclination of $26^{\circ}$, which is appropriate to the present discussion.

The wavelength dependence of the surface brightness is of critical importance in determining the ring parameters. The principal data relevant to this problem are the observations in $B$ and $V$ of Franklin and Cook (1965), the relative spectral reflectivity measurements of ring B by Lebofsky et al. (1970), the similar data from Irvine and Lane (1973) which were deduced from observations of the combined light of the Saturn system, and some recent spectral scans by Kharitonova and Teifel (1973). The data are in reasonable agreement for $\lambda \lesssim 6000 \AA$ if we bear in mind the color dependence of the opposition effect as reported by Franklin and Cook and Irvine and Lane. At longer wavelengths, however, there are some serious disagreements, which might reflect differences in inclination angle of the rings during the observations or possibly a temporal variation. We shall limit ourselves to the observations by Lebofsky et al. as the most direct and completely reported results at this time. Using their data to scale the visual reflectivity, we obtain in the $B$ band $R_{B}^{0} \simeq 0.83$ and a maximum value near $1 \mu$ of $R_{R}^{0} \simeq 1$. . These data do not include a differential opposition effect. Since the differential opposition effect between the blue and the visual is approximately 5\% (Franklin and Cook, 1965; Irvine and Lane, 1973), we shall take $R_{B}^{0}=0.87$. We shall for the present neglect any differential opposition effect between the visual and the red, in spite of the indication for such an effect from Irvine and Lane. We note that the peak reflectivity in the red is much higher in the results of Irvine and Lane and Kharitonova and Teifel than that given by Lebofsky et al.

We wish our theoretical model to match both the absolute brightness measurements and the shape of the phase curves in $B$ and $V$. We may facilitate this comparison by considering the diagram in Figure 5. The vertical axis represents the primary scattered radiation, including the shadowing effect, computed at $\alpha=0.094^{\circ}$. This will be given theoretically by $a \Phi(\alpha=0.094)\langle S(\alpha=0.094)\rangle$, where we recall that this value of $\alpha$ is the minimum obtained during the observations of Franklin and Cook. The horizontal axis in Figure 5 represents the sum of the higher order scattering, which according to the model is $\sum_{n=2}^{\infty} R_{n}$. The dashed curves designated $R, V$ and $B$ are the loci of points which satisfy the observed absolute brightness in the red, visual, and blue, respectively. For agreement with the model the absolute brightness must be

$$
R(\alpha=0.094)=a \Phi(\alpha=0.094)\langle S(\alpha=0.094)\rangle+\sum_{n=2}^{\infty} R_{n}(a, \Phi),
$$

where the indicated arguments draw attention to the dependence of the quantities on phase angle $\alpha$ and phase function $\Phi$. We have plotted as an example the lower observational limits of the absolute surface brightness in $V$ and $R$, and have shown an uncertainty of \pm 0.05 for $B$ as an example of the possible uncertainty in these measurements. In fact, these lower limits on the observed brightness allow the largest possible range of particle albedo $a$ in the comparison with theory, and also lead to a lower limit on the volume density $D$. 


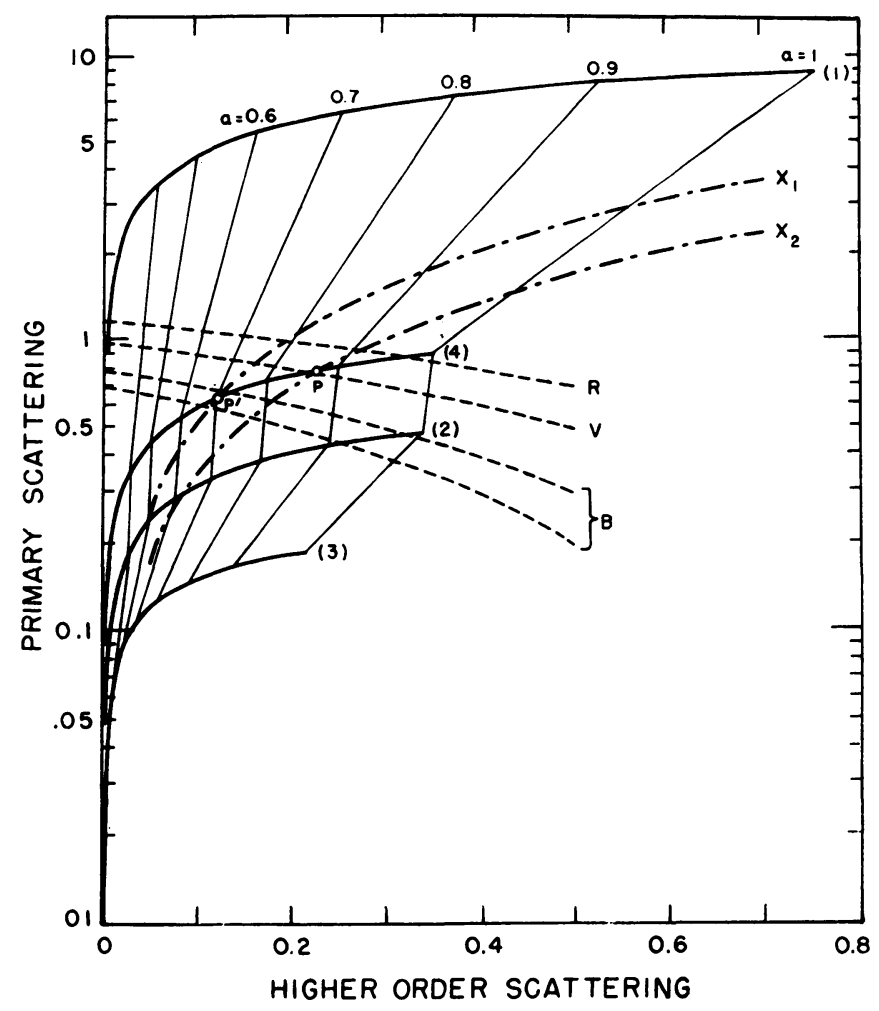

Fig. 5. Diagram for comparison of observation and theory. Vertical axis gives primary scattering contribution to total brightness, horizontal axis gives multiple scattering contribution. See text.

TABLE I

Multiple scattering contribution $x$ for $\tau=1$

$\begin{array}{ll}D=0.012 & \\ B & 0.14 \pm 0.02 \\ V & 0.26 \pm 0.02 \\ D=0.010 & \\ B & 0.17 \pm 0.02 \\ V & 0.29 \pm 0.02 \\ D=0.008 & \\ B & 0.22 \pm 0.22 \\ V & 0.34 \pm 0.02\end{array}$

For given $D$ and $\tau$, the overall shape of the phase curve $M(\alpha)$ depends principally upon the fraction of multiple scattering $x$. By experimenting with a wide choice of values for these parameters and also for the phase function $\Phi$ and the single scattering albedo $a$, we find that the sharp peak in the opposition effect depends primarily upon the value of $D$, and that the observations restrict $D$ to a narrow range around the value 


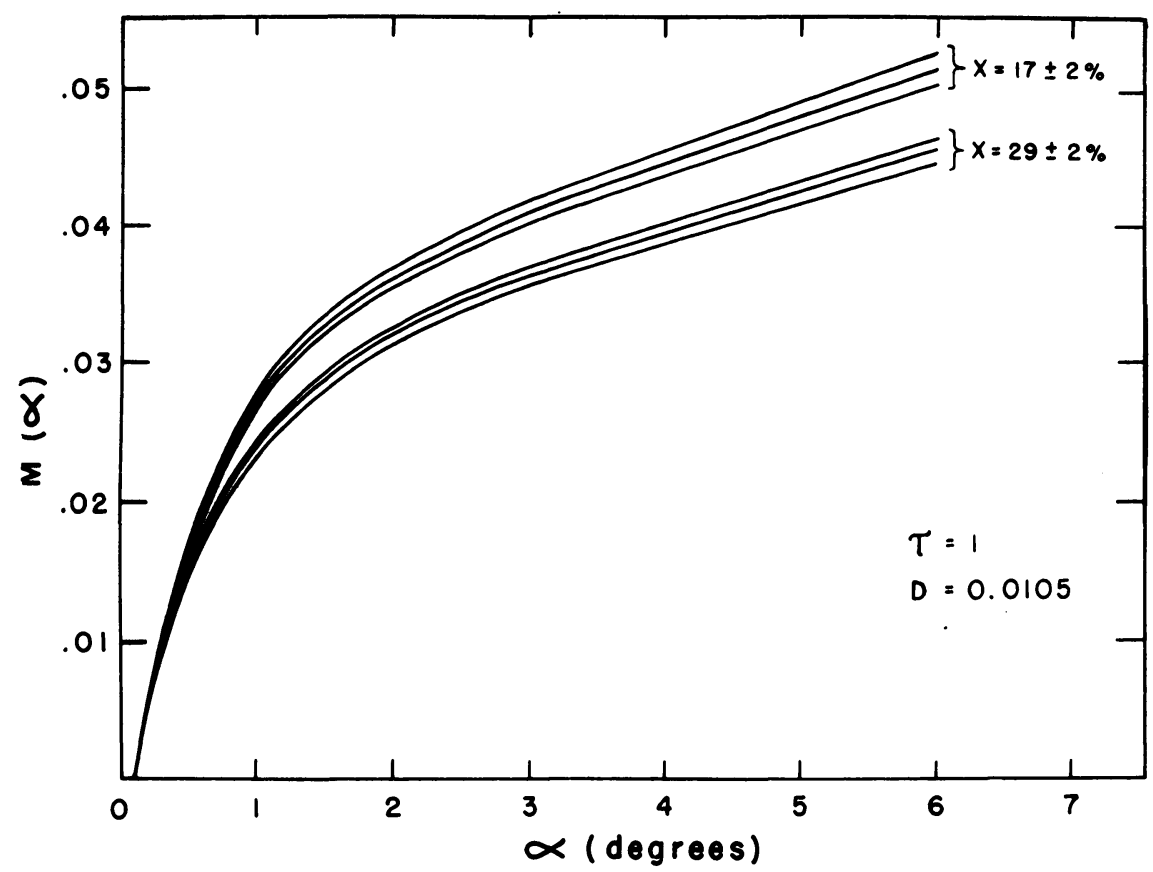

Fig. 6. Theoretical phase curves which match the observations of Franklin and Cook (1965). Fraction of multiple scattering is $x$, other parameters are shown. Note that $\theta=\pi-\alpha$.

0.01 . Let us for the present take $\tau=1$ on the basis of the observations of stellar occultations by the rings discussed by Cook et al. (1973). We then list in Table I values $x$ for several choices of $D$ which produce theoretical phase curves according to Equation (11) which agree with the observations in $B$ and $V$. For example, for $D=0.010$, the appropriate values of $x$ are 0.29 and 0.17 in $V$ and $B$, respectively. The dash-dot curves plotted in Figure 5 are now the loci of points for which the fraction of multiple scattering is $x_{1}=0.17$ and $x_{2}=0.29$, respectively. The corresponding phase curves are shown in Figure 6.

We may now use Figure 5 to determine the single scattering albedo $a$ and properties of the phase function $\Phi$ for the ring particles if we assume that the differences in brightness and phase curve for $B$ and $V$ result only from a change in particle albedo $a$. In other words, we assume that as the albedo of the particles changes with wavelength, the relative angular distribution of scattered light remains unchanged. This is a reasonable approximation for large, bright particles (which are necessary to produce the shadowing effect and the observed high ring brightness) for which geometrical optics is valid. Our whole approach to the shadowing effect through geometrical optics also requires that $\tau$ be independent of wavelength. If we now call the intersection between the curves $V$ and $x_{2}$ in Figure 5 a point $P$, and the intersection between the middle of the range $B$ and the curve $x_{1}$ a point $P^{\prime}$, then the theoretically computed brightness curve for the rings which passes through both the points $P$ and $P^{\prime}$ will 
match the observed absolute brightness and also the observed phase curves. We have plotted in Figure 5 such theoretical brightness curves for four different phase functions obtained from Equation (22). We have taken the center of the Sun and Earth in directions corresponding to the Franklin and Cook observations. The solid lines represent such brightness curves for the phase functions (1) $b=0, g_{1}=0, g_{2}=-0.7$ (a very strong backward peak with no forward scattering); (2) $b=1, g_{1}=0, g_{2}=0$ (iso-

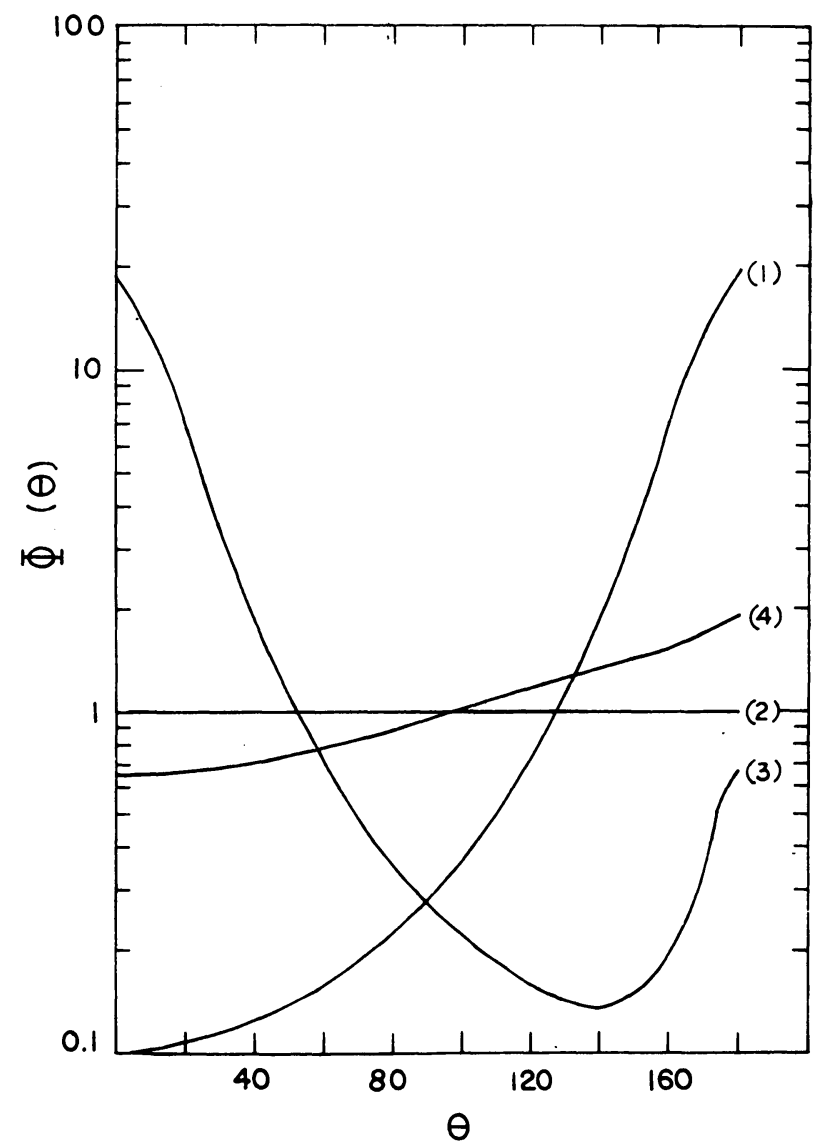

Fig. 7. Four sample phase functions $\Phi$ (see Equation (22)). Parameters described in the text.

tropic scattering); (3) $b=0.988, g_{1}=0.7, g_{2}=-0.805$ (a very strong forward peak with a small backward peak, reminiscent of the phase function for terrestrial clouds); (4) $b=0.995, g_{1}=-0.14, g_{2}=-0.84$ (a more slowly varying backward scattering phase function with a slight peak near $180^{\circ}$ ). These phase functions are illustrated in Figure 7. All of them except (2), isotropic scattering, have a similar slope near $180^{\circ}$ which produces a roughly satisfactory shape to the phase curve in the linearly varying region. The albedo $a$ is the only variable unspecified in the theoretical brightness 
curves in Figure 5, and it thus serves as a parameter whose variation along the curves is indicated.

The power of this procedure is illustrated by the large differences between the curves (1-4) in Figure 5. The requirement that the model match both the shape of the phase curve and the absolute brightness clearly puts significant restrictions on the form of the phase function. In particular, it is quite evident that neither the phase function with a very strong backward peak nor that with a very strong forward peak can match the observations. Some degree of backscatter is required to match the phase curve, so that the phase curve must be similar in shape to the curve (4). Although the shape of the phase curve (apart from the opposition peak) depends principally on the values of $\Phi$ near $180^{\circ}$ (corresponding to the small phase angles observable for Saturn), an appropriate phase function cannot be very different from the curve (4). If it decreased much more sharply with decreasing $\theta=\pi-\alpha$, it will not satisfy the normalization condition. The addition of a shallow forward peak to the phase function would be possible and would require a lower backward peak; that is, the phase function would become more isotropic.

We may now determine the single scattering albedo from the position of the points $P$ and $P^{\prime}$ on the curve (4) in Figure 5. We find $a_{V}=0.87$ and $a_{B}=0.70$. By normalizing the phase function (4) to unity at $\alpha=180^{\circ}$ and integrating, we may obtain the phase integral $q$ for the ring particles as $q=2.1$. The resulting geometric albedos in the visual and blue for the ring particles are then $p_{V}=a_{V} / q=0.41$ and $p_{B}=0.33$. The shape of the particle phase function is compared to those for the Moon and for a Lambert surface in Figure 8. The shape is quite similar to the Moon near $\alpha=0$, but the ring particle brightness falls off less rapidly with increasing $\alpha$ than does that of the

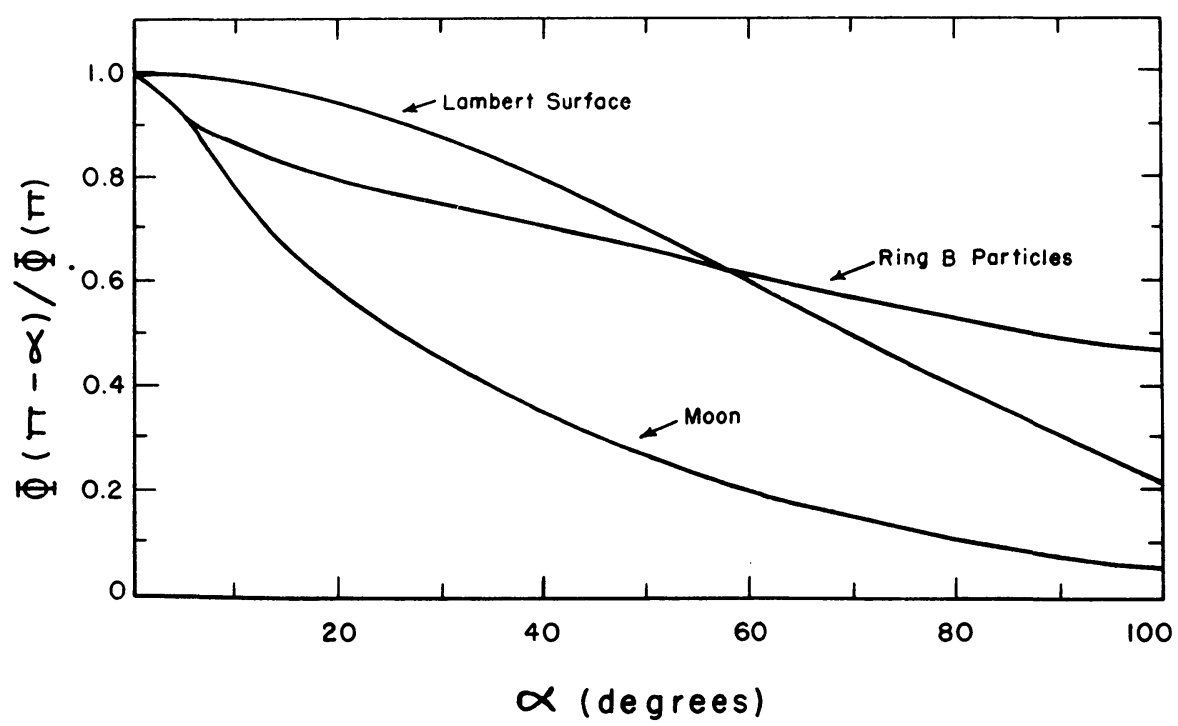

Fig. 8. Ring particle phase curve $\Phi(\pi-x) / \Phi(\pi)$ compared with lunar phase curve from Rougier (1934) and with phase curve for Lambert sphere. 
Moon. This is in agreement with the results of Veverka (1973) for snow covered objects.

\section{Allowable Range of Parameters}

As a result, we may say that a satisfactory model of the $B$ ring which matches the observed phase curves in $B$ and $V$ and also the corresponding absolute brightnesses has optical thickness $\tau=1$, a volume density $D=0.010$, and ring particles with a phase function given by (4) in Figure 7 and Bond albedos of $a_{V}=0.87$ and $a_{B}=0.70$.

It is of course important to see how much each of these parameters can be varied without disrupting the fit of the model to the data. Because the parameters cannot be varied independently if the model is going to continue to match the observations, the problem is difficult. Some possible directions in which changes may occur are sketched below.

If the optical thickness $\tau$ of the layer is kept constant at a value of unity, the value of $D$ can be reduced only slightly from that discussed above. When $D=0.008$, computations (whose results are shown in Table I) indicate that the multiple scattering contributions $x$ needed to match the phase curves are $x_{1}=0.22(B)$ and $x_{2}=0.34(V)$. Extrapolation of the curve connecting the new points $P^{\prime}$ and $P$ in this case shows that the curve will cross the dashed curve $R$ when the particle albedo in the red $a_{R} \simeq 1.0$. In other words, lowering of $D$ must be compensated by an increase in multiple scattering which requires an increase in the particle albedo in $V$ and $B$, and a resulting increase in the particle albedo for $R$ also. But $a_{R} \leqslant 1$ on physical grounds, so that the brightness of the rings in the red could not be matched by the model if $D \leqslant 0.008$. It might be expected from Figure 3 that reduction of $D$ to a value less than 0.005 would begin to reduce the shadowing effect. This is in fact true, but the resulting shape of the opposition effect is too steep to agree with the observations.

As has been pointed out in a previous section, if the value of $D$ is too large, the opposition peak will be too broad. For the present choice of the other parameters the upper limit on $D$ is approximately 0.012 . With this value we obtain multiple scattering contributions $x$ of 0.26 in $V$ and 0.14 in $B$. The corresponding values of albedo parameterizing the theoretical brightness curve are $a_{V}=0.82$ and $a_{B}=0.65$. The phase function in this case will be slightly more backward scattering than the phase function (4).

We may summarize these results by saying that, for $\tau=1$ and the minimum surface brightness allowed by the observations

$$
\begin{aligned}
& 0.008<D<0.012 \\
& 0.65<a_{B}<0.75 \\
& 0.82<a_{V}<0.9 \\
& 0.93<a_{R}<1.0 .
\end{aligned}
$$

We have also examined the case $\tau=0.7$ as an estimate of the effects of this possible lower limit for ring $B$. We find that a somewhat smaller value of $D \simeq 0.006$ is required to match the shape of the opposition peak. The magnitude of the shadowing effect due to single scattering decreases with decreasing $\tau$, but this may be compensated by 
decreasing the dilution of the shadowing effect due to multiple scattering. In the present case we find it necessary to take approximately $x(V)=0.21$ and $x(B)=0.10$. Constructing the figure analogous to Figure 5 for the present case we find that a somewhat more backscattering phase function is required, although in general its shape will be similar to that for the case $\tau=1$. The corresponding albedos are found to be $a_{V}=0.85$ and $a_{B}=63$, which are not drastically different from their values for $\tau=1$. The value of $\tau$ cannot be much less than 0.7 , since lower values do not produce a sufficiently large opposition effect in the ultraviolet (cf. Irvine and Lane, 1973).

To investigate the effects of choosing a larger optical thickness $\tau_{0}$, we have also carried out computations for $\tau=2$. In this case we find $D \approx 0.013$ and multiple scattering contributions of $x(V)=0.40$ and $x(B)=0.28$. These larger fractions of multiple scattering are necessary to dilute the larger shadowing effect produced by primary scattering as $\tau$ increases. The albedos are slightly increased relative to $\tau=1$, the new values being $a_{V} \approx 0.90$ and $a_{B} \approx 0.78$, while the corresponding phase function has less of a backward peak than for $\tau=1$.

In addition to the lower limit for the absolute surface brightness $R_{V}^{0}$, we must investigate the effect on the model of choosing the apparent mean observational value $R_{V}^{0} \simeq 1.1$. The range of $D$ and $x$ which match the phase curves remains unchanged, since the phase curves measure only relative brightness. The particle albedos are increased, but the requirement that $a_{R} \leqslant 1$ in the red provides also an upper limit on $a_{V}$ and a lower limit on $D$ (through interaction with $x$ ). As a result, the allowable range of particle albedo is reduced relative to that for a lower absolute surface brightness. We find $0.85 \leqslant a_{V} \leqslant 0.90$ and $0.66 \leqslant a_{B} \leqslant 0.72$ for $\tau=1$. The corresponding phase function has approximately a $10 \%$ larger backward peak than in the previous case, so that the phase integral becomes $g \simeq 1.95$ instead of 2.1 .

\subsection{Distribution OF PARTICLE SIZES}

Up to this point we have assumed that the ring particles can be characterized by a single effective radius $\varrho$. Within the framework of this assumption we can determine that size if we know the geometric thickness of the rings $t$. From the definitions of the optical depth $\tau=\pi \varrho^{2} n t$ and $D=(4 / 3) \pi \varrho^{3} n$ we find that

$$
\varrho=\frac{3 D}{4} \frac{D}{\tau} t
$$

The ring thickness has been measured by Kiladze (1969) and Focas and Dollfus (1969) to be approximately $2 \mathrm{~km}$. Taking $\tau \simeq 1, D \simeq 0.01$ and $t \simeq 2 \mathrm{~km}$, we find that $\varrho \simeq 15 \mathrm{~m}$. This size is consistent with the recent radar results obtained by Goldstein and Morris (1973).

The above result is quite deceptive, however. To see this we must investigate the possible influence on our results of allowing for a distribution in particle sizes. Let us assume that the number of particles with radii between $\varrho$ and $\varrho+\mathrm{d} \varrho$ is given by

$$
\mathrm{d} n=f(\varrho) \mathrm{d} \varrho
$$


per unit volume. If we assume that the volume density $D$ remains small enough so that the small element of area $\varepsilon$ on a test particle in Figure 2 shielded from the Sun and Earth by a particle in the range $\varrho \rightarrow \varrho+\mathrm{d} \varrho$ is independent of the probability that it is shielded by a particle of any other radius, we may write for the first order reflected intensity including the shadowing effect

$$
\begin{aligned}
R_{1}^{s}=\left(\frac{a \Phi}{4 \mu \mu_{0}}\right)\left(\pi \int_{\varrho_{1}}^{\varrho_{2}} f(\varrho) \varrho^{2} \mathrm{~d} \varrho\right) \times \int_{0}^{t} \exp \left[-h^{\prime}\left(\frac{1}{\mu}+\frac{1}{\mu_{0}}\right) \times\right. \\
\\
\left.\times\left(\pi \int_{\varrho_{1}}^{\varrho_{2}} f(\varrho) \varrho^{2} \mathrm{~d} \varrho\right)+\int_{\varrho_{1}}^{\varrho_{2}} f(\varrho) C\left(\varrho, h^{\prime}\right) \mathrm{d} \varrho\right] \mathrm{d} h^{\prime},
\end{aligned}
$$

where the upper and lower limits on particle size have been labeled $\varrho_{2}$ and $\varrho_{1}$. The rest of the theory remains the same.

The choice of possible forms for the particle distribution function is of course infinite. Bobrov has investigated the relation

$$
f(\varrho)=K \varrho^{-s},
$$

where $K$ is a constant and $s$ is a parameter describing the shape of the distribution. This distribution law is common in meteor astronomy, and of course can lead to a predominant number of quite small particles.

We have investigated the effect of a uniform particle distribution $(s=0)$ and also the cases $s=2$ and 3 . In all cases we find that the results are quite insensitive to the lower limit $\varrho_{1}$ of the particle size distribution provided that $\varrho_{1} \lesssim 1 \mathrm{~cm}$, but do remain quite dependent upon the value of $D$. This shows that the shadowing effect in the case when a dispersion of particle sizes is present continues to determine the volume density $D$ quite precisely, but that the mean particle size remains uncertain provided that it is large enough to produce the required geometrical optics shadowing.

On the other hand, the size distribution cannot be too sharply varying. When the parameter $s=3$ in Equation (34), it is not possible to obtain agreement with the observations. For a value $s=2$, the range of permissible values for the volume density $D$ is somewhat increased $(0.005 \lesssim D \lesssim 0.013)$.

For $s=0$ the results are quite similar to the monodisperse case. If we fix the optical depth $\tau=1$ and the geometric thickness $t=2 \mathrm{~km}$, the value of $D$ will be a function of the upper limit $\varrho_{2}$ of the size distribution. To obtain the necessary value of $D \approx 0.012$, we must have $\varrho_{2} \lesssim 25 \mathrm{~m}$. The mean particle size is then $\langle\varrho\rangle \approx 12 \mathrm{~m}$.

The corresponding upper limit of the particle size distribution when $s=2$ is $15 \mathrm{~m} \leqslant$ $\leqslant \varrho_{2} \leqslant 50 \mathrm{~m}$. The range of permissible values of the multiple scattering contribution $x$ is also increased, so that $0.17 \leqslant x_{V} \leqslant 0.30$ and $0.05 \leqslant x_{B} \leqslant 0.18$. Using the same method as in the monodisperse case (cf. Figure 5), we find the allowable range for the particle albedo to be $0.72 \leqslant a_{V} \leqslant 0.82$ and $0.42 \leqslant a_{B} \leqslant 0.6$ for $R_{V}^{0}=1.0$. The upper limit on the mean particle size is estimated to be less than $2 \mathrm{~m}$. 


\subsection{Tilt EFfECT}

We may test the validity of the type of model chosen here by comparison with other types of observation. Particularly important are the data on the surface brightness as a function of solar illumination angle $\theta_{0}$ (tilt angle). Observations over a limited range of $\theta_{0}$ have been made by Camichel (1958) and Focas and Dollfus (1969). More accurate and homogeneous measurements have been published by Price (1973), although this data includes both rings together. At $\theta=\theta_{0}=64^{\circ}$, the mean surface brightness of ring B is $20 \%$ greater than the mean of A and B (Franklin and Cook, 1965; Camichel, 1958). As a first approximation towards removing the effect of ring $A$, we may assume that this ratio applies also at other $\theta_{0}$. Price's data so corrected are plotted in Figure 9, together with Camichel's results scaled to agree with Price at $\theta_{0}=26^{\circ}$. The corresponding theoretical curve was computed for $\tau=1, a_{V}=0.9$, and the phase function (22)

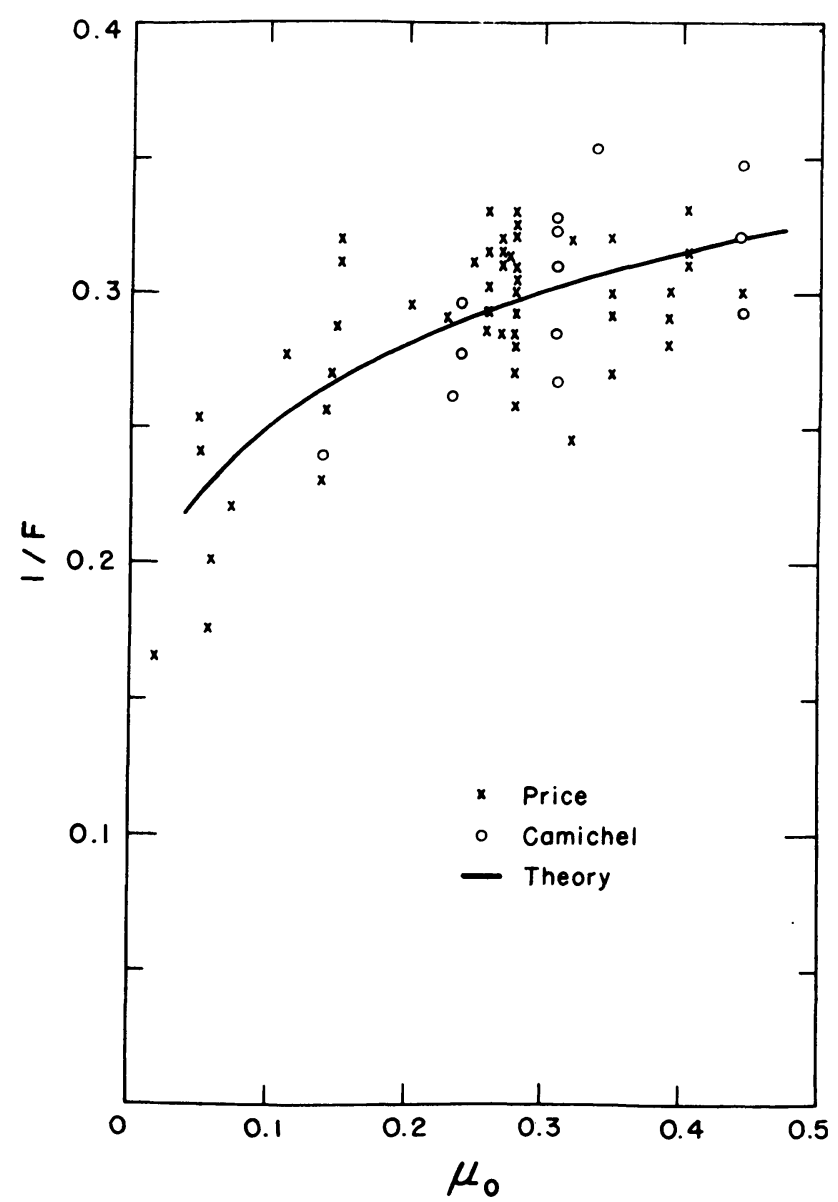

Fig. 9. Surface brightness $\mu_{0} R\left(\alpha=6^{\circ}\right)$ for ring $\mathrm{B}$ as a function of solar illumination angle arccos $\mu_{0}$ for the $V$ band. Theoretical curve computed for $\tau=1, a_{V}=0.9$, and the phase function noted in the text. 
with $b=0.995, g_{1}=-0.16$, and $g_{2}=-0.85$. This form of $\Phi$ has $q=1.95$ and fits the observed phase curves and their mean absolute surface brightness $R_{V}^{0}=1.1$.

The agreement between theory and observation is quite good. This result clearly illustrates the importance of multiple scattering in the rings, since the surface brightness for primary scattering alone would decrease with increasing $\theta_{0}$, in opposition to the observations and to the multiple scattering model.

\section{Conclusion}

The models of the rings described here match the observed phase curves, absolute surface brightness, and tilt effect for ring B. They prescribe a value of the volume density $D$ close to 0.01 and a phase function which is somewhat backscattering (of the general form shown by curve 4 in Figure 7). The maximum range for the particle Bond albedos in the visual and blue are

$$
\begin{aligned}
& 0.72 \leqslant a_{V} \leqslant 0.90 \\
& 0.42 \leqslant a_{B} \leqslant 0.75 .
\end{aligned}
$$

It is not possible to obtain a reliable estimate of the mean particle size without a knowledge of the particle size distribution, but it appears that $\left\langle\varrho^{2}\right\rangle^{1 / 2} \lesssim 15 \mathrm{~m}$.

\section{Acknowledgements}

We are grateful to Drs J. Cuzzi, D. Van Blerkom, F. Franklin, and R. Murphy for several stimulating discussions. This research was supported in part by NASA Grant NGL 22-010-023.

\section{References}

Bobrov, M. S.: 1970, Solar System Res. 4, 127 (English transl.).

Camichel, H.: 1958, Ann. Astrophys. Paris 21, 231.

Cook, A. F., Franklin, F. A., and Palluconi, F. D.: 1973, Icarus 18, 317.

Focas, J. and Dollfus, A.: 1969, Compt. Rend. Acad. Sci. Paris 268, 100.

Franklin, F. A. and Cook, A. F.: 1965, Astron. J. 70, 704.

Goldstein, R. M. and Morris, G. A.: 1973, Icarus 20, 260.

Hansen, J. E.: 1969, Astrophys. J. 155, 565.

Irvine, W. M.: 1964, Bull. Astron. Inst. Neth. 17, 266.

Irvine, W. M., 1966, J. Geophys. Res. 71, 2931.

Irvine, W. M. and Lane, A. P.: 1973, Icarus 18, 171.

Kharitonova, G. A. and Teifel, V. G.: 1973, Astron. Tsirk., No. 747.

Kiladze, R. I.: 1969, Abastumani Astrophys. Obs. Bull., No. 37, 151.

Lebofsky, L. A., Johnson, T. V., and McCord, T. B.: 1970, Icarus 13, 226.

Pierce, A. K. and Waddell, J. H.: 1961, Mem. Roy. Astron. Soc. 68, 89.

Price, M. J.: 1973, Astron. J. 78, 113.

Rougier, G.: 1934, Astronomie 48, 220; 48, 281.

Seeliger, H.: 1887, Abhandl. Bayer. Akad. Wiss., Math.-Naturn., Kl. II 16, 405-516.

Seeliger, H.: 1895, Abhandl. Bayer. Akad. Wiss., Math.-Naturn., Kl. II 18, 1.

Van de Hulst, H. C.: 1948, Astrophys. J. 107, 220.

Van de Hulst, H. C.: 1957, Light Scattering by Small Particles, John Wiley \& Sons, Inc., New York, p. 106. 
Van de Hulst, H. C. and Irvine, W. M.: 1963, La physique des planètes, 11 th Liège Astrophys. Symp., p. 78.

Veverka, J.: 1973, Icarus 20, 304.

\section{DISCUSSION}

Smith: Does your model take into account the recent radar results from Goldstein, which appear to require very large (preferably meters if not even tens of meters) particles?

Irvine: It is reassuring that our model results in particle sizes which are consistent with the radar results. In my mind the most puzzling question at the moment is the lack of a definitive passive radio detection of the rings.

Feigelson: I doubt that it is possible to apply radiative transfer equations to Saturn ring particles, which are relatively large, if the optical density is low.

Irvine: The optical thickness of the rings is of the order of unity. If the geometric thickness is a couple of kilometers, and the particle sizes are in the meter range, I think a multiple scattering procedure is reasonable. Of course if the particles have radii in the kilometer range, or the rings are a monolayer, the situation is different.

Bobrov: I have some comments. (1) Several important parameters of Saturn's rings are poorly known; for instance, optical thickness of B-ring, phase curve of A-ring, etc. The C-ring is practically unexplored. This means that, for progress in research, further observations are needed.

(2) The mutual shadowing effect theory supposes the rings to be a system many particles thick, which faces very serious dynamical difficulties. Possibly the extremely sharp peak of the rings' phase curve results from the scattering by single particles. To clear up this question we need laboratory photometry down to $1^{\circ}$ phase.

(3) One must also not forget that up to now we know only a small portion of the rings' phase curve $0-6^{\circ}$, and therefore the observation of the phase variation of the rings from space vehicles may add sufficient information to our knowledge. 